\title{
CMEARTICLE
}

\section{Clinics in diagnostic imaging (178)}

Raymond Chung ${ }^{1}$, MBBS, FRCR, Ashish Chawla ${ }^{1}$, MD, DABR, Wilfred CG $\underline{\text { Peh }}^{1}$, FRCP, FRCR

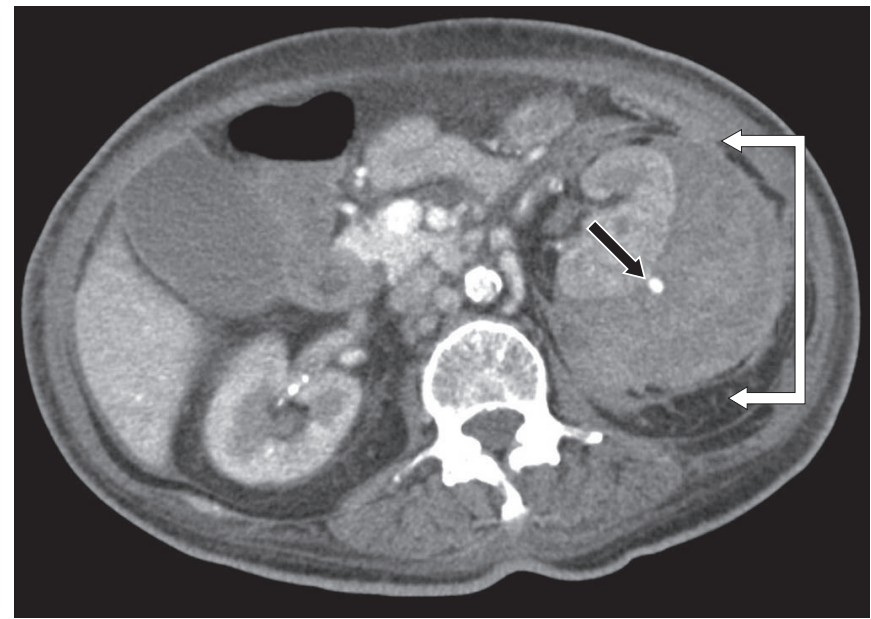

Fig. 1 Axial contrast-enhanced CT image of the abdomen taken at the level of the kidneys.

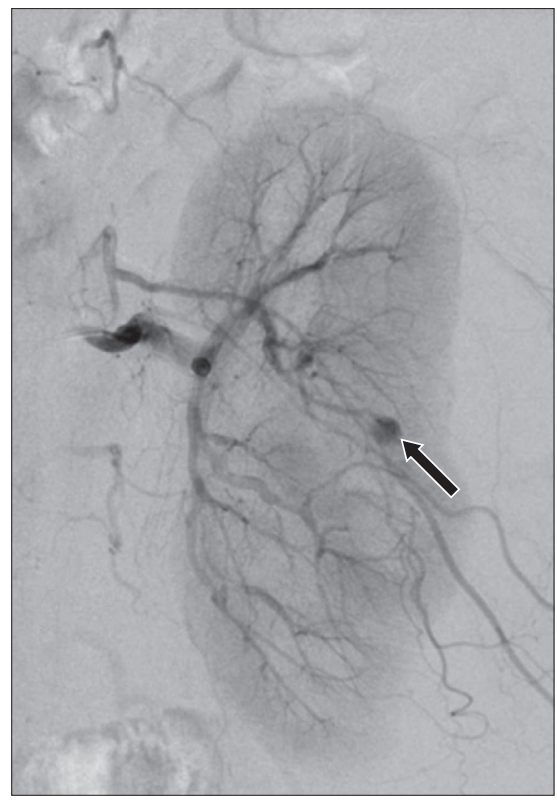

Fig. 2 Digital subtraction angiogram of the left renal artery.

\section{CASE PRESENTATION}

A 64-year-old woman with end-stage renal failure secondary to diabetic nephropathy was referred to the emergency department from the dialysis unit following intradialytic hypotension and giddiness. On further questioning, she described a four-day history of dull, non-radiating left flank pain. There was no history of trauma or iatrogenic injury such as renal biopsy.

On examination, the patient had a palpable left flank mass. Admission laboratory serum tests revealed profound anaemia: haemoglobin $4.7 \mathrm{~g} / \mathrm{dL}$ (normal range [NR] 11.5-15.0 g/dL); haematocrit $14.8 \%$ (NR $36.0 \%-46.0 \%$ ); and platelet count $320 \times 10^{9} / \mathrm{L}\left(\mathrm{NR} 130-400 \times 10^{9} / \mathrm{L}\right)$. Clotting screen was deranged: prothrombin time 11.4 seconds (NR 9.2-11.0 seconds); activated partial thromboplastin time $>190.0$ seconds (NR 23.4-36.6 seconds); and international normalised ratio 1.12. Urgent enhanced computed tomography (CT) of the abdomen and subsequent digital subtraction angiography (DSA) were performed (Figs. 1 \& 2). What do the CT and DSA images show? What is the diagnosis? 

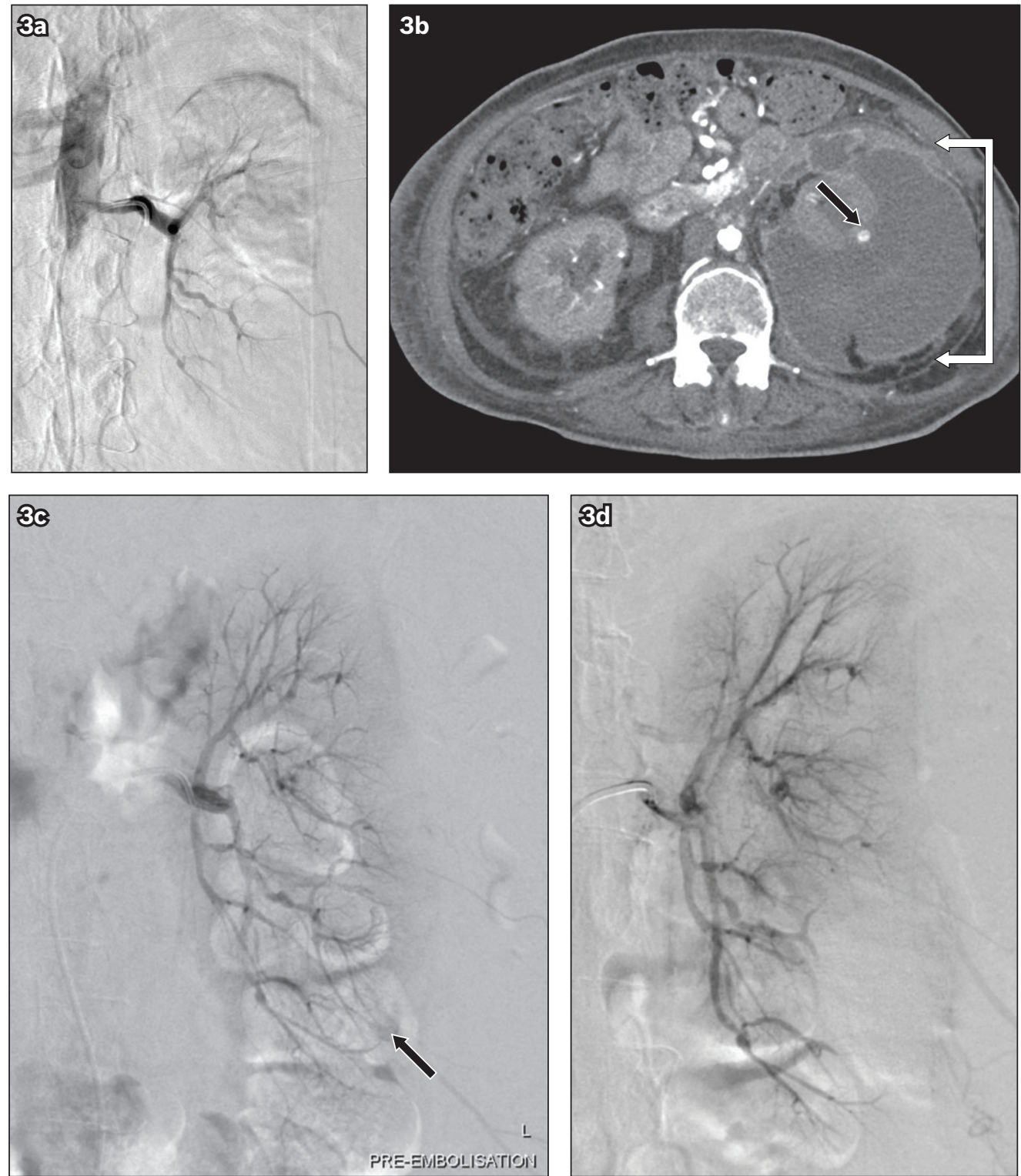

Fig. 3 (a) Digital subtraction angiogram (DSA) of the left renal artery after the first embolisation shows no further perfusion of the pseudoaneurysm. (b) Two weeks later, axial contrast-enhanced CT image of the abdomen shows an enlarging subcapsular haematoma with a thick, enhancing wall (white arrows) and a cortical lower pole pseudoaneurysm (black arrow). (c) Pre-embolisation DSA confirms the findings and shows late filling of the cortical lower pole pseudoaneurysm (arrow), while (d) post-embolisation DSA shows no further perfusion of the inferior pole pseudoaneurysm.

\section{IMAGE INTERPRETATION}

Contrast-enhanced CT image of the abdomen shows a large left renal subcapsular haematoma (white arrows) with a 6-mm cortical pseudoaneurysm (black arrow) (Fig. 1). The haematoma displaces the kidney anteriorly with expansion of the renal fascia. No underlying mass lesion is present. DSA (Fig. 2) confirms a solitary pseudoaneurysm (arrow) arising from the distal arcuate and cortical branches of the posterior division of the left renal artery in an interpolar position.

\section{DIAGNOSIS}

Wünderlich syndrome and pseudoaneurysm.

\section{CLINICAL COURSE}

Following the catheter-directed angiograms, selective pseudoaneurysm embolisation was successfully performed
(Fig. 3a). The patient remained haemodynamically stable after the procedure with no further reduction in haemoglobin levels and was subsequently discharged 11 days later. She was readmitted four days later due to confusion. This was initially treated as bacteraemic sepsis caused by extended-spectrum beta-lactamases (ESBL) producing Escherichia coli (E. coli), presumably from the large subcapsular haematoma. Contrastenhanced CT of the abdomen confirmed no further filling of the embolised pseudoaneurysm, stable haematoma size and no new sites of haemorrhage.

Two weeks following the re-admission, the patient had an episode of symptomatic hypotension. Repeat contrast-enhanced CT (Fig. 3b) demonstrated a new 5-mm cortically placed lower pole pseudoaneurysm with an increase in size of the haematoma, which was consistent with a repeated spontaneous haemorrhagic episode. The pseudoaneurysm that was previously embolised 


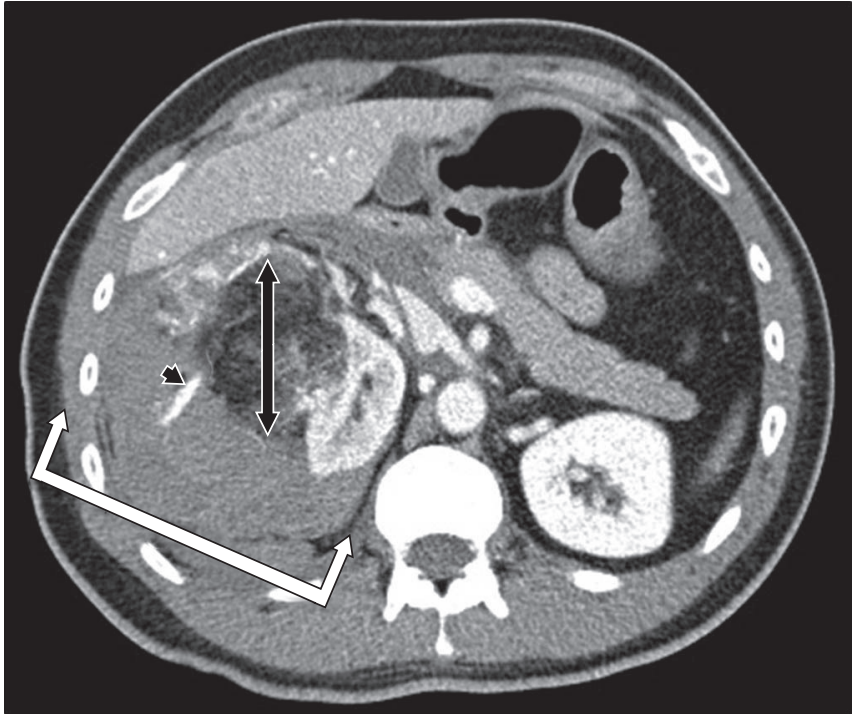

Fig. 4 A 40-year-old man with haemorrhagic rupture of a 6.3-cm right renal angiomyolipoma. Axial contrast-enhanced CT image of the abdomen shows a large right retroperitoneal haematoma (white arrows) secondary to active extravasation (black arrowhead) from a large angiomyolipoma; the latter is diagnosed by its characteristic imaging components of mature adipose tissue, smooth muscle and aberrant vasculature (black arrow).

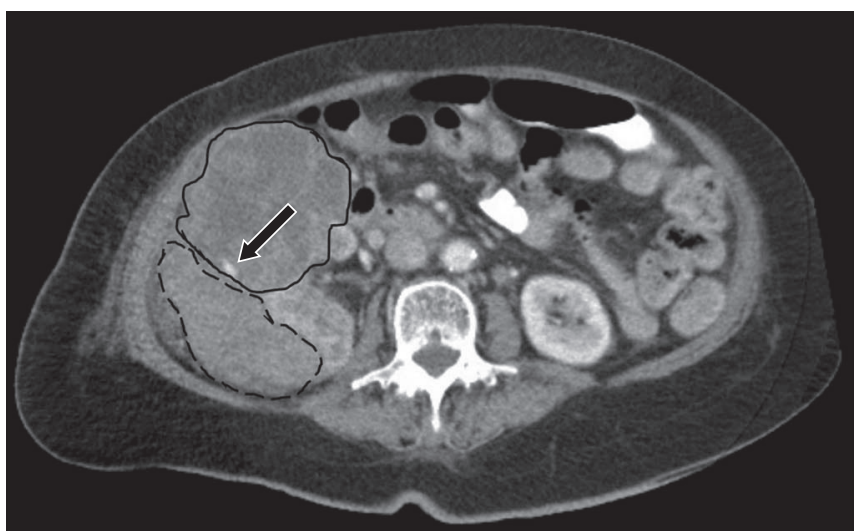

Fig. 5 A 78-year-old woman with haemorrhagic rupture of a large RCC. Axia contrast-enhanced CT image of the abdomen shows an anterior mass with mixed soft-tissue enhancement and central necrosis (solid outline) with site of active arterial extravasation (arrow) and secondary mixed attenuating haematoma posteriorly (dashed outline).

remained occluded. DSA confirmed the CT findings (Fig. 3c) and the interventional radiologist again proceeded to successfully embolise the pseudoaneurysm (Fig. 3d). Repeat CT five days later confirmed technical success with the absence of pseudoaneurysm perfusion.

However, two months later, due to worsening ESBL-producing E. colibacteraemia, the patient underwent open nephrectomy and renal bed washout, which confirmed a large-volume, purulent haematoma. Histological examination of the nephrectomy specimen reported large perinephric and renal capsule abscesses. There was no focal, underlying renal parenchymal mass, or any histological evidence of vasculitis. Postoperatively, recurrent infected haematomas were observed in the renal bed, tracking along the pericolic gutter and into the pouch of Douglas. Following multiple percutaneous drain insertions and prolonged active drain management, the collections finally resolved after three months.

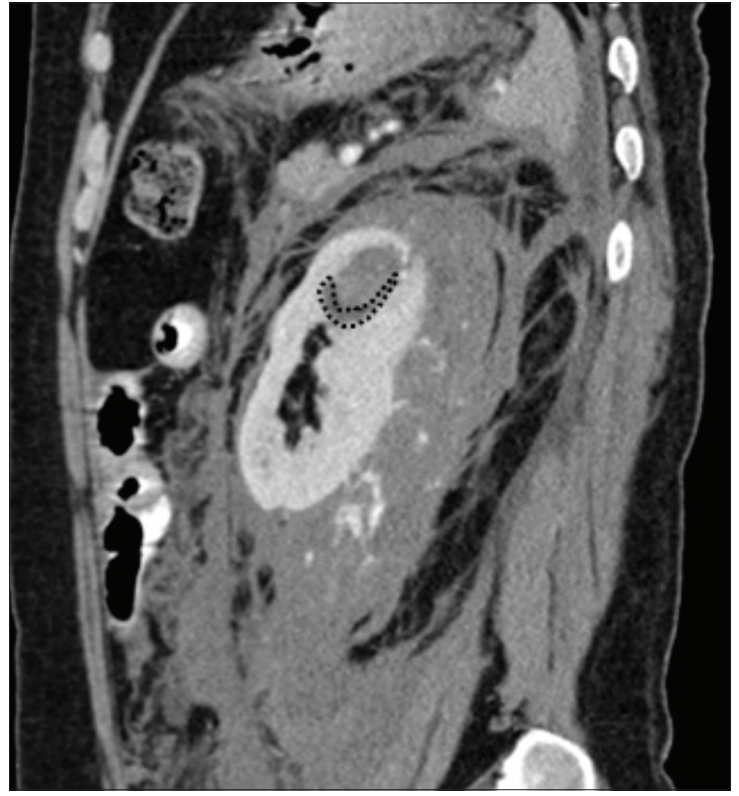

Fig. 6 A 39-year-old man with haemorrhagic rupture of a renal cyst. Sagittal contrast-enhanced CT image of the abdomen shows a faint inferior hypodense rim of the bleeding endophytic renal lesion, consistent with an underlying haemorrhagic cyst (dotted margin).

\section{DISCUSSION}

Wünderlich syndrome, defined as spontaneous, non-traumatic renal haemorrhage into the subcapsular space, is a rare entity that was first reported in $1856^{(1)}$ and can only be found in small case series in the literature. ${ }^{(2,3)}$ Patients classically present with the Lenk's triad of flank pain, palpable mass and hypovolaemic shock. Spontaneous haemorrhage is most commonly attributed to renal tumours such as angiomyolipomas (AMLs; Fig. 4), vasculitides or infection. Less common aetiologies include blood dyscrasias and anticoagulation.

Imaging plays a vital role to confirm the diagnosis and identify any underlying pathological process that may result in a predisposition to haemorrhage. $\mathrm{AML}$, the most common benign renal tumour associated with spontaneous haemorrhage, especially in lesions that are larger than $4 \mathrm{~cm}$, is a perivascular epithelioid cell tumour consisting of varying proportions of mature adipose tissue, smooth muscle and aberrant vasculature. On CT, it is characteristic but not pathognomonic for AMLs to be visible as macroscopic fat-containing tumours (Fig. 4); rarer differentials include fat-containing renal cell carcinoma (RCC) and liposarcoma. Lipid-poor AMLs can also make diagnosis difficult and may require additional use of unenhanced pixel analysis techniques to identify small quantities of fat. ${ }^{(4)}$ Classically, on magnetic resonance imaging, the tumour shows variable highsignal return on non-fat-saturated T1 - and T2-weighted sequences, with loss of signal on fat-saturated sequences.

RCC is the malignant tumour that is most frequently associated with spontaneous haemorrhage, with features of central necrosis and heterogeneously enhancing solid soft-tissue components. The most common subtype of RCC, clear cell RCC, is usually heterogeneously hypervascular in the arterial phase. ${ }^{(5)}$ It may be difficult to discern ill-defined enhancing soft tissue from adjacent heterogeneously hyperdense haematoma (Fig. 5), especially if the 
tumour is small. Spontaneous haemorrhage from an existing cyst is more readily diagnosed with the aid of preceding imaging, as the haematoma and active extravasation are likely to obscure the underlying cyst. Otherwise, a slim residual rim of hypodensity within the circular nidus of the haematoma and extravasation may implicate a cyst as the underlying pathology (Fig. 6).

In the event that there is no discernible aetiology, follow-up CT is recommended to exclude an underlying neoplasm masked by the large-volume haematoma. In our case, the aetiology of the first spontaneous haemorrhage was attributed to the patient's clotting dyscrasia secondary to heparin use during dialysis, with resulting prolonged prothrombin and activated partial thromboplastin time. The development of a second pseudoaneurysm that was metachronous, anatomically distinct and cortical-based, after a gap of approximately one month, was postulated to have arisen from cortical stripping by the haematoma and subsequent tearing of the cortical arteries. To the best of our knowledge, no previous cases of successfully treated Wünderlich syndrome have resulted in a second delayed haemorrhagic event due to the formation of a new, anatomically distinct pseudoaneurysm. Therefore, despite the confirmed technical success of a transarterial embolisation procedure, it is prudent to maintain a high level of suspicion for a metachronous bleeding site in patients with symptomatic hypotension and recurrent loin pain.

Despite the potential morbidity and mortality of Wünderlich syndrome, it is seldom within the admitting clinician's list of differential diagnoses. ${ }^{(6)}$ Nevertheless, arterial phase CT is the recommended imaging modality of choice ${ }^{(2)}$ and usually illustrates the diagnosis by revealing a heterogeneously dense subcapsular haematoma with or without a site of bleeding, such as a circular enhancing pseudoaneurysm. However, rarer causes, such as segmental arterial mediolysis, may not be differentiated. Simple episodes of renal subcapsular haemorrhage, in the absence of haemodynamic compromise or pseudoaneurysms, can be managed expectantly. The presence of a pseudoaneurysm, as in this case, requires treatment irrespective of size and symptomatology. Lack of structural support for the damaged arterial wall results in a higher propensity to rupture. Endovascular techniques are increasingly the preferred approach, regardless of aetiology, given their minimally invasive nature, shorter recovery period and ability to preserve renal parenchyma function..$^{(7,8)}$ The precise endovascular treatment, whether it is embolisation with coils, liquid embolics or stent graft exclusion, varies according to the anatomical characteristics of the aneurysm and operator experience. In any case, the primary aim is to occlude or exclude flow to the pseudoaneurysm while maintaining maximal perfusion to the remaining renal parenchyma. ${ }^{(7)}$ Surgery may be required for haemorrhage control or haematoma evacuation in those with haemodynamic instability or uncontrolled sepsis, respectively. ${ }^{(2,6,9)}$ This case of recurrent Wünderlich syndrome was initially managed successfully by transarterial embolisation. However, superadded infection of the large subcapsular haematoma warranted nephrectomy and renal bed washout two months later.

In conclusion, Wünderlich syndrome refers to spontaneous, non-traumatic renal haemorrhage into the subcapsular and perirenal spaces. Despite its severity, it is rarely considered as a clinical diagnosis, but can be confirmed on CT in virtually all cases. Transarterial and open surgical techniques remain complementary in the often challenging management of this condition.

ABSTRACT Wünderlich syndrome is a rare entity characterised by spontaneous retroperitoneal haemorrhage with renal origin. We present a case of Wünderlich syndrome secondary to clotting dyscrasia in a 64-year-old woman. The patient experienced a second Wünderlich haemorrhagic event with metachronous pseudoaneurysm formation, which was likely secondary to the large subcapsular haematoma stripping the renal capsule and tearing the cortical arteries. Selective pseudoaneurysm embolisations were successfully performed on both occasions. This clinical entity, its imaging differential diagnoses and management are discussed.

Keywords: pseudoaneurym embolisation, renal haemorrhage, retroperitoneal haemorrhage, Wünderlich syndrome

\section{REFERENCES}

1. Albi G, del Campo L, Tagarro D. Wünderlich's syndrome: causes, diagnosis and radiological management. Clin Radiol 2002; 57:840-5.

2. Molina Escudero R, Castillo OA. Spontaneous retroperitoneal haemorrhage of renal origin (Wunderlich syndrome): analysis of 8 cases. Arch Esp Urol 2013; 66:925-9. English, Spanish.

3. Wang BH, Pureza V, Wang H. A tale of Wünderlich syndrome. J Surg Case Rep 2012; 2012(11).

4. Jinzaki M, Silverman SG, Akita H, et al. Renal angiomyolipoma: a radiological classification and update on recent developments in diagnosis and management. Abdom Imaging 2014; 39:588-604.

5. Pedrosa I, Sun MR, Spencer M, et al. MR imaging of renal masses: correlation with findings at surgery and pathologic analysis. Radiographics 2008; 28:985-1003.

6. Sierra-Díaz E, Belmonte-Hernández MV, Villanueva-Pérez MA, García-Gutiérrez M. [Non-traumatic spontaneous retroperitoneal bleeding: the effect of an early and accurate diagnosis]. Cir Cir 2015; 83:206-10. Spanish.

7. Chung R, Touska P, Morgan R, Belli AM. Endovascular management of true renal arterial aneurysms: results from a single centre. Cardiovasc Intervent Radiol 2016; 39:36-43.

8. Jain V, Ganpule A, Vyas J, et al. Management of non-neoplastic renal hemorrhage by transarterial embolization. Urology 2009; 74:522-6.

9. Chiong E, Consigliere D, Esuvaranathan K. Renal tumours: a common incidental finding. Singapore Med J 2007; 48:495-502; quiz 503. 


\section{SINGAPORE MEDICAL COUNCIL CATEGORY 3B CME PROGRAMME} (Code SMJ 201706B)

Question 1. Wünderlich syndrome is a potentially life-threatening condition that classically presents with:

(a) Chest pain and breathlessness.

(b) Loin pain and palpable mass.

(c) Haematuria.

(d) Hypovolaemic shock.

Question 2. Regarding Wünderlich syndrome:

(a) Neoplastic causes are the most common, among which the tumour most frequently resulting in spontaneous haemorrhage is renal cell carcinoma.

(b) Angiomyolipomas (AMLs) are the most common benign tumours that cause spontaneous renal haemorrhage.

(c) Blood dyscrasias are associated with the syndrome.

(d) It is diagnosed predominantly on clinical grounds with little role for imaging investigations.

Question 3. Regarding the appearance of AMLs on computed tomography (CT):

(a) AMLs are hypervascular with mixed areas of soft tissue and central necrosis.

(b) It is characteristic, but not pathognomonic, of AMLs to appear as fat-containing renal tumors.

(c) AMLs consist of mature fat, smooth muscle and aberrant vasculature, and can only be diagnosed on CT when all components are visibly identifiable.

(d) Larger AMLs have a higher chance of spontaneous haemorrhage compared to smaller ones.

Question 4. Regarding imaging investigation of Wünderlich syndrome:

(a) Arterial phase contrast-enhanced CT is the imaging investigation of choice.

(b) All patients require direct catheter angiography to identify potential pseudoaneurysms.

(c) Follow-up imaging is required to exclude an underlying neoplasm masked by the initial subcapsular and perirenal haematoma.

(d) Cross-sectional imaging with MR imaging may help characterise an underlying lesion following haemorrhage.

Question 5. Regarding management of Wünderlich syndrome:

(a) Haemodynamically unstable patients can be managed conservatively in the absence of initial imaging findings of haemorrhage and infection.

(b) Only pseudoaneurysms larger than $4 \mathrm{~cm}$ require treatment, as smaller lesions are unlikely to have complications.

(c) In the presence of sepsis, infected perinephric haematomas should be evacuated.

(d) Endovascular techniques in haemostasis form the mainstay of primary treatment in haemodynamically stable patients.

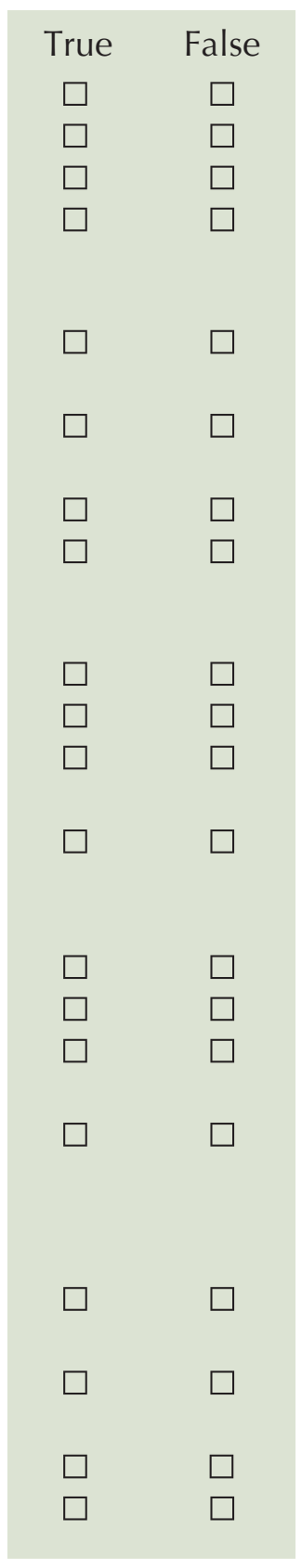

\footnotetext{
Doctor's particulars:

Name in full

MCR number

Specialty:

Email address

\section{SUBMISSION INSTRUCTIONS:}

(1) Visit the SMJ website: http://www.smj.org.sg/current-issue and select the appropriate set of questions. (2) Provide your name, email address and MCR number. (3) Select your answers and click "Submit".

\section{RESULTS:}

(1) Answers will be published online in the SMJ August 2017 issue. (2) The MCR numbers of successful candidates will be posted online at the SMJ website by 6 August 2017. (3) Passing mark is $60 \%$. No mark will be deducted for incorrect answers. (4) The SMJ editorial office will submit the list of successful candidates to the Singapore Medical Council. (5) One CME point is awarded for successful candidates.

Deadline for submission: (June 2017 SMJ 3B CME programme): 12 noon, 27 July 2017.
} 